\title{
Discussion on the Process Dimension by Tracking Chart Method
}

\author{
Shuping Lv \\ Dalian Vocational \& Technical College, Dalian, China \\ 964511001@qq.com
}

Keywords: process size; process margin; process size tracking; dimensional chain

\begin{abstract}
In the machining process of parts, the process dimension is calculated one by one using the process dimension chain calculation, that is called single-chain calculation. That is, a process dimension chain diagram is calculated once. This single-chain calculation method is only applicable to parts with fewer processes. If processes is large, the parts that do not overlap or the reference are converted multiple times, and the calculation of the process size is also a single-chain calculation method, which will be complicated and cumbersome, also it will make mistakes often. Once the error occurs, it needs to be reworked. The overall process sizes are also linked to each other. In the actual process dimension chain calculation, all or most of the rework calculations often occur. Therefore, the overall contact calculation (ie, the dimension tracking chart method) for all process dimensions of the part can avoid errors. So this paper will illustrate the establishment and calculation of the dimension tracking chart by way of examples.
\end{abstract}

\section{Tracking Chart Format Establishment}

The process dimension tracking chart method is the overall calculation method. It uses economical machining accuracy to calculate the process size and process margin, and takes into account the overall relationship between the process dimensions. The process dimension tracking chart method is to draw all the process dimensions on a chart to visually find the route of the error transfer process and to easily calculate the process size and process margin. This charting method also makes it easy to use a computer for auxiliary calculations.

(1) Draw a part outline above the chart;

(2) In the center of the chart, align with the outline of the part, draw the dimension tracking chart according to the program content, and "." in the figure shows the starting point of the process size, the surface indicated by the arrow is the working surface, and the process dimensions is indicated by a straight line connecting " $"$ " and the arrow, and draw the location standard for each process;

(3) On the right side of the dimension tracking chart, draw the machining process number, the process content, and the process size basic dimensional tolerance;

(4) On the right side of the dimension tracking chart, draw the serial number of the machining process, the basic margin and tolerance of the process margin;

(5) Draw the design dimensions at the bottom right of the dimension tracking chart;

(6) In the middle of the dimension tracking chart, align with the outline of the part, and draw “._." to indicate the design dimensions.

\section{Determination of Process Dimensions and Tolerances}

Firstly, to determine the basic size of each process. The design size corresponding to the basic size of the process means that the size limit at both ends of the process basic size corresponds to the bottom design size from the top to the bottom.

The calculation and determination of process dimensional tolerances is the basis of the entire chart method calculation process. To determine the tolerance of the process size must meet two principles, as follows:

First, the determined tolerances shall not exceed the tolerances required on the drawings and shall ensure that the tolerances of the final machining result dimensions meet the design 
requirements;

Second, the tolerance of each process size should conform to the economical type of processing in this process, which is beneficial to reduce the processing cost.

According to these two principles, the tolerances of the dimensions of each process are initially determined item by item (refer to the "Economic Accuracy of Dimensional Deviation" in the technician manual), and the table is filled in from the bottom to the top according to the symmetrical labeling form.

And then calculate the tolerance of the process size, which is the tolerance obtained by the mutual transfer of the relevant dimensional errors during the machining process. Therefore, to calculate the tolerance of the process size, the dimension tracking rule is needed to find the route of the error transmission, that is, the size connection. Dimensional tracking rule: Find the arrow from the two ends of the calculated process size, find the arrow and turn the arrow to the starting point of the process size, and then continue to find the arrow until you find the tracking route at both ends meet at the starting point of a certain process size, then stop.

Fill the error transmission route found by the dimensional tolerance of each process into the chart, and form a ring by the tolerance of the process size found by tracking, to calculate the dimension tolerance.

\section{Determination of Process Margin and Tolerance}

The principle of calculating the process margin is that the process margin is sufficient and reasonable, especially to ensure that the minimum of the process margin is sufficient to eliminate various factors.

Surface quality of the upper process;

Process dimensional tolerance of the previous process;

Position error of the previous process;

Installation error of the workpiece in the process etc.

Firstly, to calculate the tolerance of the process dimension, secondly to calculate the tolerance of process margin, in order to accurately understand the influence of the tolerance of the determined process dimension on the process margin, so as to ensure that the process margin is sufficient and reasonable. The process margin is generally the amount of indirect control, and the tolerance of the process margin depends on the cumulative result of the process dimension tolerance transfer. Therefore, the tolerance for calculating the process margin is calculated one by one from bottom to top according to the size tracking rule.

\section{Example}

The specific example is used to illustrate the establishment and calculation method of the tracking dimension chart.

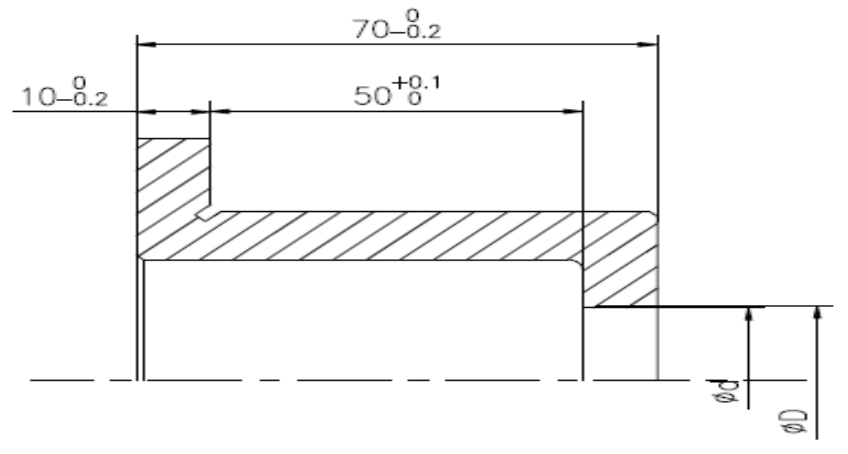

Fig. 1 Bushing design as drawing

Figure 1 shows a simplified design of the bushing. The axial dimension of the bushing is shown in the upper part and the diameter is in the range of $\Phi 80-\Phi 120 \mathrm{~mm}$. It is divided into six processes, and the process dimensions are shown respectively in the process diagram as shown in Figure 2. 
The machining allowances and tolerances of the end faces can be found in the relevant manual. (Refer to the "Margin" section in the Technician's Manual), to fill in the table from the bottom to up.
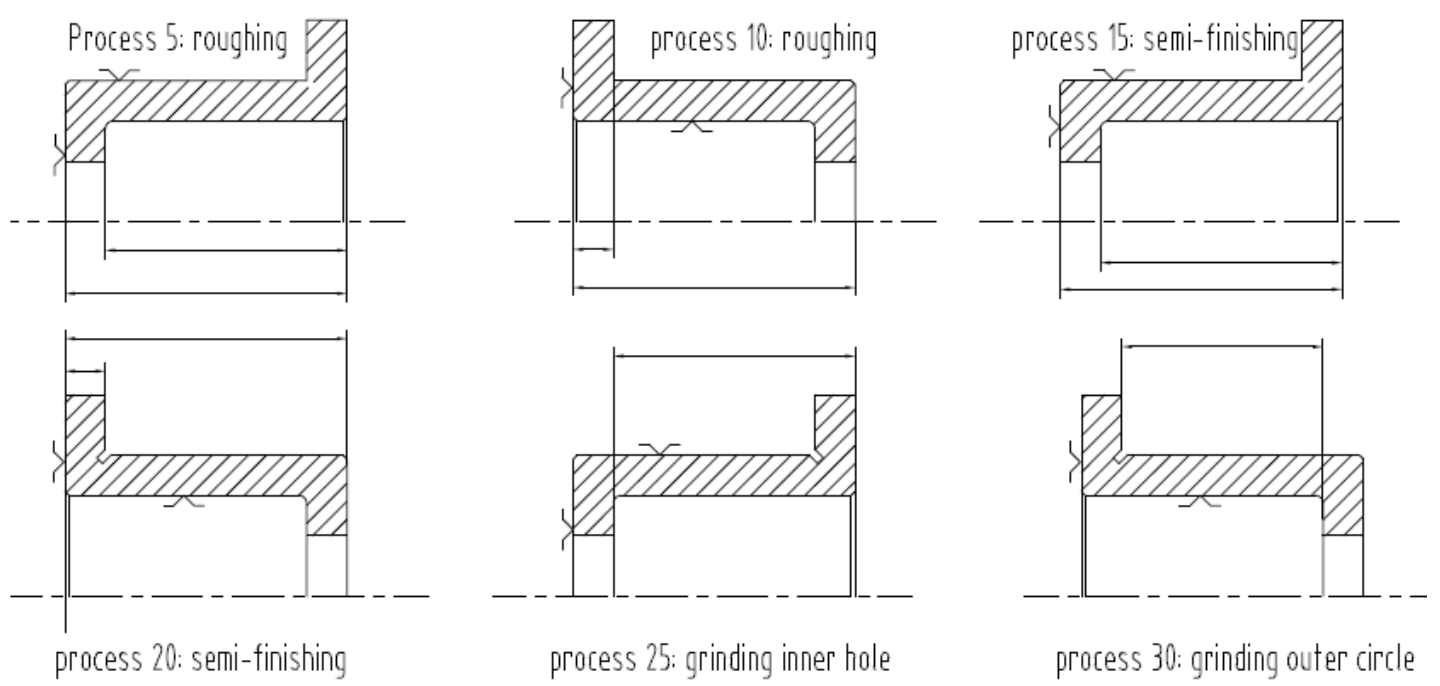

Fig. 2 Bushing process route

The steps for establishing the tracking size chart are as follows:

\subsection{Draw a Schematic Diagram of the Axial Section of the Part.}

The surface lines are drawn from the respective axial surfaces in the figure, and the surfaces are numbered ( $A, B, C, D$ in the figure). The design dimensions of these surfaces are shown in the upper part of the diagram as shown in Fig. 3.

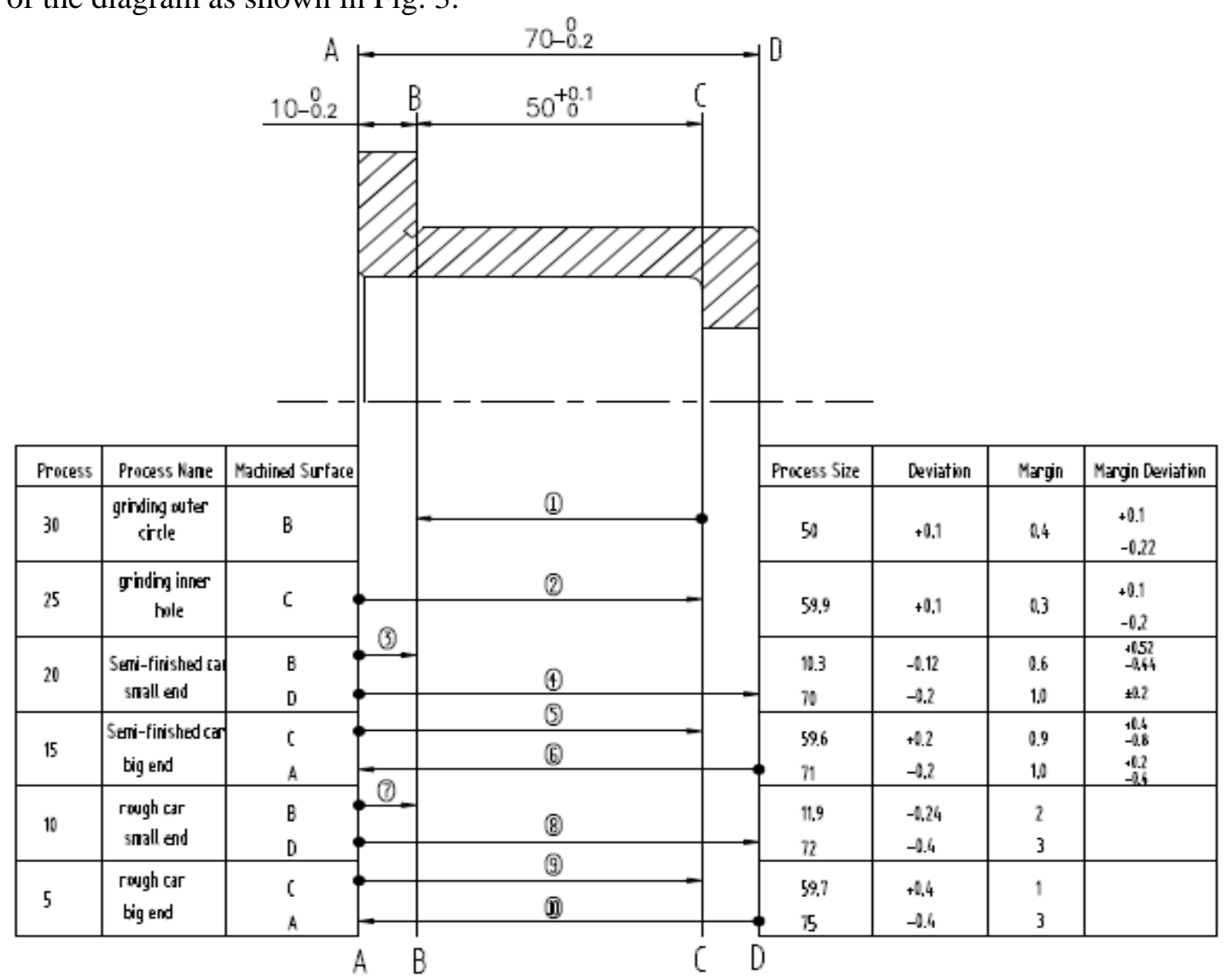

Fig.3 Bushing tracking chart 


\subsection{Draw Process Dimension.}

According to the determined process route, the list process number is arranged from the back to the front on the lower side of the diagram. In one process, if there are several surfaces to be processed, the post-processing is placed on the top, and the first processing is placed on the bottom, arranged in this order.

Draw the process dimensions of the machined surface according to the process standard selected in the process specification, and draw a point at the process reference. At the surface to be machined, all process dimensions are numbered for the convenience of the following description. The figure shows from top to bottom, from (1) to (10).

The margin is not indicated in the figure, so when the same surface is machined in different processes, the arrows of the machined dimensions point to the same surface line. This is the case for the dimension line (4) in the process 20 and the dimension line (8) in the process 10.

Fill in the table with the remaining value of each machined surface in the process (Note: the general margin value is the basic margin, and the allowance will have the largest and smallest change due to machining tolerances)

Determine the basic size value of each process size, method as follows:

Size (1): upward both sides of (1), meet with design size $50_{0}^{+0.1} \mathrm{~mm}$, so the basic size of Size (1) is $50 \mathrm{~mm}$.

Size (2): upward both sides of (2), there is no direct design size between $A$ and $C$, it is $60 \mathrm{~mm}$ according to $10_{-0.2}^{0} \mathrm{~mm}$ and $50_{0}^{+0.1} \mathrm{~mm}$.

Size (3): lead of Size (3) meet $10_{-0.2}^{0} \mathrm{~mm}$ on the surface $B$, and meet arrow of size (1).This arrow indicates that the surface is to be processed once, so the machining allowance (refer to the margin $Z_{1}$ of size (1) at $10 \mathrm{~mm}$ plus the arrow should be taken as the basic size of size (3), that is: $10+0.3=10.3 \mathrm{~mm}$

Other basic dimensions are analogized by the above method. When both ends of the lead wire meet the arrow, the machining allowance of the surface indicated by each arrow should be added to the relevant design size as the basic size of the process. That is:

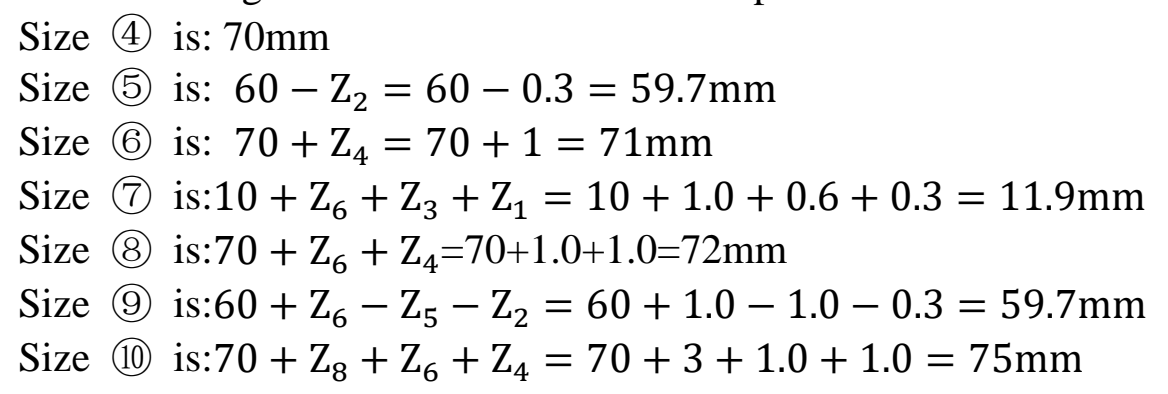

\subsection{Determine the Dimensional Tolerances and Margin Tolerances of Each Process.}

The tolerances of the dimensions of the various processes associated with the design dimensions are determined first, for which the relevant dimension chain must be found. The principle of listing the dimension chain in the dimension chart is as follows

When obtaining the process size related to a certain design size, the dotted line is drawn from both ends of the size. After entering the process size area, the arrow that encounters the process size should be turned along the size to the process reference point. After that, the downward lead is continued until the dotted line drawn from the ends of the closed loop is combined with one end of a process dimension line. This process is also called "tracking" method.

\subsubsection{Process Dimensional Tolerance Determination.}

Dimensions $10_{-0.2}^{0} \mathrm{~mm}$ are drawn from the two sides of $A$ and $B$ with a dotted line. When entering the process size area, the arrow of (1) is encountered. The arrow of (1) is reversed to the starting point of process size (1) and then the downward lead is continued. (2) meets the extension 
line along the A side. The dimensions (1), (2) included in the above-mentioned dotted line are the constituent rings of the process size chain which ensures the design size $10_{-0.2}^{0} \mathrm{~mm}$. In the dimensional chain drawn by the tracking method, the process dimensions (1) and (2) are constituent rings, and the design dimensions $10_{-0.2}^{0} \mathrm{~mm}$ are closed loops. According to the principle that the sum of the component ring tolerances is equal to the closed ring tolerance, the tolerance values of the design dimensions can be appropriately assigned to the process dimensions (1) and (2), that is, the sum of the tolerances of the process dimensions (1) and (2) cannot be greater than the tolerance of $10_{-0.2}^{0} \mathrm{~mm}$.

Size (1) , it can ensure directly $50_{0}^{+0.1} \mathrm{~mm}$, so the size and tolerance of (1) is: $50_{0}^{+0.1} \mathrm{~mm}$.

Size (2), Size (2) and (1) should ensure timely $10_{-0.12}^{0} \mathrm{~mm}$, so tolerance of size (2) should be calculated by dimensional chain (Fig. 4).

So

$0=\mathrm{ES}_{60}-0$

So

$$
\mathrm{ES}_{60}=0
$$

So

$$
\begin{gathered}
-0.2=\mathrm{EI}_{60}-0.1 \\
\mathrm{EI}_{60}=-0.1
\end{gathered}
$$

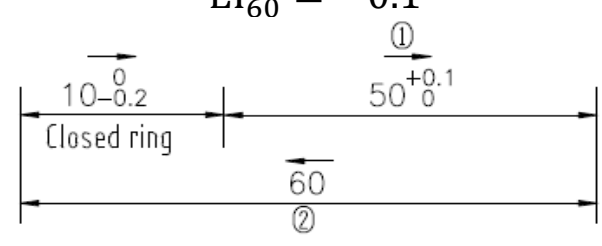

Fig. 4 Dimensional chain of size 2

Therefore, Size (2) is $60_{-0.1}^{0} \mathrm{~mm}$, Changed to the "into body" tolerance form $59.9_{0}^{+0.1} \mathrm{~mm}$.

Size(3), there is no direct relation with design size, according to the economic precision, the end face of the finished car is 6 grades. Therefore, the dimensional tolerance of size (3) is $10_{-0.12}^{0} \mathrm{~mm}$.

Size(4), (4) can ensure directly $70_{-0.2}^{0} \mathrm{~mm}$, so the size and tolerance of (4) is $70_{-0.2}^{0} \mathrm{~mm}$.

The sizes (5) and (6) are intermediate processes, which are determined according to the economic precision of the semi-finished car, respectively is $59.6_{0}^{+0.2} \mathrm{~mm}, 71_{-0.2}^{0} \mathrm{~mm}$.

Size (7), is roughing machining, process tolerance is $0.24 \mathrm{~mm}$, it is $11.9_{-0.24}^{0} \mathrm{~mm}$.

Size (8), (9), (10) are all roughing machining, process tolerance is $0.4 \mathrm{~mm}$, respectively is $72_{-0.4}^{0} \mathrm{~mm}, 59.7_{0}^{+0.4} \mathrm{~mm}, 75_{-0.4}^{0} \mathrm{~mm}$.

\subsubsection{Process Margin Tolerance Determination.}

The calculation of the margins of sizes (3) and (5) is representative, so the following is taken as an example. The tolerances for dimensions (1) and (2), (4), (6) are omitted.

The margin of the size (3), the process size that affects its change is (6), (7), (8) dimensional chain as shown in Figure 5.

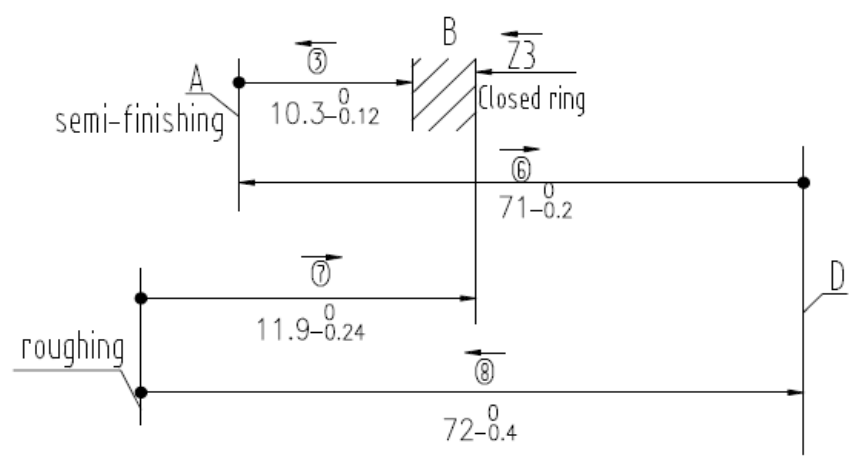

Fig.5 Margin dimensional chain of Size 3 
After calculation, $Z_{3}=0.6_{-0.44}^{+0.52} \mathrm{~mm}$.

Margin of Size(5) : Dimensional chain as Fig.6.

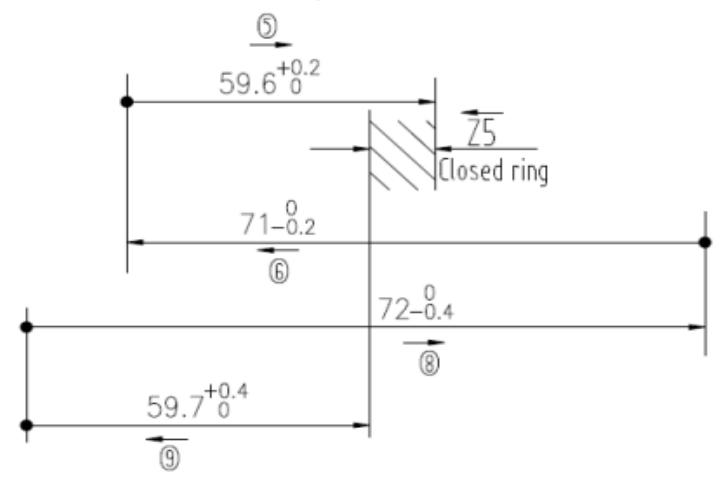

Fig.6 Margin dimensional chain of Size 5

After calculation, $Z_{5}=0.9_{-0.8}^{+0.4} \mathrm{~mm}$.

In the process of drafting the process route, it is not necessary to check the margin of each process. For example, the margin of the roughing process is large, and often it is not necessary to check the calculation.

Finally, the process dimensions, tolerances, etc. are filled in the tracking chart. As original record during process size design.

\section{Conclusion}

In a large number of mass production, if a certain surface of a part is processed through several processes, such as many processes, the benchmarks do not coincide in the process, the size has to be converted, the process size and its tolerance are more complicated to determine, in order to solve similar problems, the process size chart method is very convenient.

\section{References}

[1] Shao Wei. Formulation of mechanical manufacturing process regulations. Beijing: Mechanical Industry Press, 2011.

[2] Chang Tongli. Mechanical Manufacturing Technology and Learning. Beijing: Qinghua University Press, 2010

[3] Zhu Huanchi. Mechanical Manufacturing Technology. Beijing: Mechanical Industry Press, 2012.

[4] Zheng Xiuben. Mechanical Manufacturing Technology. Beijing: Mechanical Industry Press, 2012.

[5] Wang Fengping. Mechanical Manufacturing Technology. Beijing: Mechanical Industry Press, 2012. 